\title{
Clostridium difficile Infection Among Hospitalized Chronic Hepatitis B Virus-Infected Patients in a Chinese Hospital
}

\author{
Yunbo Chen ${ }^{1}$, Qiaodi Gui ${ }^{2}$, Qiaomai $\mathrm{Xu}^{1}{ }^{1}$, Tao $\mathrm{Lv}^{1}$, Silan Gu${ }^{1}$, Ping Shen ${ }^{1}$, Jiazheng Quan ${ }^{1}$, Yunhui \\ Fang ${ }^{1}$, Guangyong $\mathrm{Ye}^{3}$ and Lanjuan $\mathrm{Li}^{4}{ }^{4}$ \\ ${ }^{1}$ State Key Lab for Diagnosis and Treatment of Infectious Diseases, The First Affiliated Hospital, School of Medicine, Zhejiang University, Hangzhou, China \\ ${ }^{2}$ Department of Clinical Laboratory, Shaanxi Provincial People's Hospital, Xi'an, China \\ ${ }^{3}$ Department of Clinical Laboratory, Women's Hospital, School of Medicine, Zhejiang University, Hangzhou, China \\ ${ }^{4}$ State Key Laboratory for Diagnosis and Treatment of Infectious Diseases, Collaborative Innovation Center for Diagnosis and Treatment of Infectious Diseases, The First \\ Affiliated Hospital, School of Medicine, Zhejiang University, Hangzhou, China \\ "Corresponding author: Qingchun Road No. 79, Hangzhou, Zhejiang, China. Tel: +86-5787236459, Email: ljli@zju.edu.cn
}

Received 2018 March 19; Revised 2018 July 31; Accepted 2018 August 01.

\begin{abstract}
Background: Liver disease represents a risk factor for Clostridium difficile infection (CDI). However, CDI predisposition and its incidence in patients with chronic hepatitis $\mathrm{B}(\mathrm{CHB})$ have not been well-characterized.

Objectives: This study aimed at determining the incidence and risk factors of CDI in CHB patients without cirrhosis.

Methods: A retrospective case-control study was conducted on hospitalized patients in a Chinese tertiary hospital between June 2010 and June 2016.

Results: A total of $105 \mathrm{CHB}$ patients without cirrhosis were included in the present study. Among these patients, 35 (33\%) patients developed hospital-acquired CDI. A total of 35 toxigenic C. difficile strains were assigned to 15 different STs by multi-locus sequence typing (MLST). Multivariate analysis indicated that prolonged hospital stay (OR: 1.045; 95\% CI: 1.006 to 1.086) and higher Charlson scores on admission (OR: 3.063; 95\% CI: 1.602 to 5.857) were independent factors for the development of CDI among CHB patients without cirrhosis.

Conclusions: A high incidence of CDI was detected in this cohort of CHB patients. Both the prolonged hospital stay and higher Charlson scores made CHB patients more susceptible to hospital-acquired CDI. Greater emphasis on infection control measures and antimicrobial stewardship in patients with CHB during hospital admission is needed.
\end{abstract}

Keywords: Infection, Hepatitis B Virus, Clostridium difficile

\section{Background}

Clostridium difficile infection (CDI) has been increasingly viewed as a major pathogen for nosocomial diarrhea since the first report on this pathogen (1). Two common toxins (toxin A and B) produced by C. difficile have been considered to induce $C$. difficile-associated diarrhea. The emergence of NAP1/BI/027, an epidemic hyper-virulent strain in North America and Europe (2), secretes a third toxin (binary toxin, CDT), which may result in poor prognosis. Clinical CDI manifestations range from mild diarrhea to toxic megacolon and pseudomembranous colitis (3). Furthermore, CDIs are responsible for costs of approximately $\$ 1.5$ billion per year in the United States (4).

Clostridium difficile infection incidence continues to increase in both the general population and patients with liver disease, and has become a significant burden to pub- lic health worldwide $(5,6)$. Old age and exposure to both antibiotics and healthcare facilities are the main risk factors for CDI (3). It has also been known that both cirrhotic patients and liver transplant recipients are at high risk of $\operatorname{CDI}(7,8)$. However, CDI incidence and risk factors for chronic hepatitis $\mathrm{B}(\mathrm{CHB})$ patients without cirrhosis have not been adequately studied. In a previous study, a disorder in gut microbiota in patients with $\mathrm{CHB}$ was detected (9), implying that CHB may have created a favorable environment for $\mathrm{CD}$ colonization and infection.

\section{Objectives}

The present study aimed at determining the incidence and risk factors of $\mathrm{CDI}$ in $\mathrm{CHB}$ patients without cirrhosis by utilizing case-control cohorts hospitalized at a Chinese ter- 
tiary hospital, and establishing recommendations on preventing and treating $\mathrm{CDI}$ among $\mathrm{CHB}$ patients.

\section{Methods}

\subsection{Ethics Statement}

The present study was approved by the Medical Ethics Committee of the First Affiliated Hospital, School of Medicine, Zhejiang University (ZYYY 2018536).

\subsection{Patients and Definitions}

Data on CDI were collected from CHB patients without cirrhosis, who were admitted between June 2010 and June 2016 to the First Affiliated Hospital, School of Medicine, Zhejiang University. The included CDI population was limited to patients, who had CDI onset at the healthcare facility (HCF), or HCF-associated (HO-HCFA) CDI. Furthermore, HO-HCFA CDI was defined as a CDI episode that occurred within 48 hours after admission, or within 28 days after discharge (10). A CDI was considered when both the C. diffcile culture and $C$. difficile toxin assay were positive in stool samples obtained from patients with diarrhea (10). Diarrhea was defined as $\geq 3$ unformed stools within 24 hours (10).

Non-cirrhotic CHB in-patients, who were $\geq 18$ years old, were included in this study. Patients with CDI relapse were excluded. The control group included non-cirrhotic CHB inpatients with C. difficile negative diarrhea, and had been hospitalized for at least 48 hours. The subjects in the control group were randomly selected from a pool of age, gender- and admission period-matched CHB in-patients with no known CDI history. The present study was approved by the Medical Ethics Committee of the First Affiliated Hospital, School of Medicine, Zhejiang University.

\subsection{Collection of Toxigenic C. difficile Isolates}

For isolating C. difficile, the stool sample was inoculated on cycloserine cefoxitin taurocholate agar (Oxoid Ltd., Cambridge, UK), supplemented with 7\% sheep blood after an alcohol shock procedure, and the strains were confirmed by matrix-assisted laser desorption-ionization time-of-flight mass spectrometry (MALDI-TOF-MS) with a Microflex LT system (Bruker Daltonik GmbH, Bremen, Germany). DNA was isolated according to a previously described method (11). All strains were tested for the presence of $t c d A, t c d B, c d t A$, and $c d t B$ genes by the polymerase chain reaction (PCR), as described by Kato et al. (11) and Stubbs et al. (12), respectively. Briefly, the PCR protocol for the toxin A gene was 35 cycles consisting of $95^{\circ} \mathrm{C}$ for 20 seconds and $62^{\circ} \mathrm{C}$ for 120 seconds. The PCR protocol for toxin $\mathrm{B}$ gene included 35 cycles of $95^{\circ} \mathrm{C}$ for 20 seconds and $55^{\circ} \mathrm{C}$ for 120 seconds. The thermal profiles for the CDTa and $C D T \mathrm{~b}$ genes were determined at 30 cycles of $94^{\circ} \mathrm{C}$ for 45 seconds, $52^{\circ} \mathrm{C}$ for 60 seconds, and $72^{\circ} \mathrm{C}$ for 80 seconds. Toxigenic C. difficile isolate was confirmed as $C$. difficile isolate positive with toxin A gene and/or toxin B gene.

\subsection{Multilocus Sequence Typing}

Multilocus sequence typing (MLST) was carried out with seven housekeeping genes ( $a d k, a t p A, d x r, g l y A, r e c A$, and tpi) for all of the isolates according to Griffiths et al. (13). The assignment of the allele number and Sequence Type (ST) was performed with $C$. difficile MLST database homepage (http://pubmlst.org/cdifficile/).

\subsection{Data Collection}

The biochemical parameters and hematological parameters (serum albumin level, serum creatinine level, serum alanine aminotransferase level, serum aspartate transaminase level, serum total bilirubin level, international normalized ratio, prothrombin time, white blood cell count, hemoglobin concentration, and platelet count) in the admission samples were recorded.

\subsection{Statistical Analysis}

Numeric variables were expressed as mean \pm standard deviation (SD) or median with interquartile range (IQR). Statistical significance was determined by Student's t-test or Mann-Whitney's U-test. Categorical variables were calculated as percentages and compared using the chi-square test. Risk factors were first screened by univariate analysis, and were further extracted by step-wise multivariate logistic regression analysis. The bivariate-resultant factors when $\mathrm{P}>0.05$ were ascertained. Logistic regression was used to determine the odds ratios with $95 \%$ confidence intervals (95\% CIs). The SPSS package (version 23.0; SPSS Inc., Chicago, IL, USA) was used for all statistical analyses.

\section{Results}

\subsection{Patient Population}

From June 2010 to June 2016, 105 CHB patients without cirrhosis were selected for the present study (Table 1). Among these patients, 35 patients were diagnosed with HO-HCFA CDI. Among all CDI patients $(n=35), 25$ patients were male (71.4\%) and their mean age was 43.6 years old. The mean hospital stay among CDI patients was 25 days. In the control group $(n=70), 50$ subjects were male $(71.4 \%)$, and their mean age was 41.7 years old. Furthermore, mean duration of hospitalization among the controls was 12 days. Two patients (5.7\%) in the CDI group and four patients (5.7\%) in the control group died during the hospital stay. 


\begin{tabular}{|c|c|c|c|c|}
\hline Patient Characteristics & Total, $\mathbf{N}=105$ & CDI, $\mathbf{N}=35$ & Control, $\mathrm{N}=70$ & P Value \\
\hline Age & $43.0 \pm 13.33$ & $43.6 \pm 14.47$ & $41.7 \pm 12.78$ & 0.49 \\
\hline Male, No. (\%) & $75(71.4)$ & $25(71.4)$ & $50(71.4)$ & 1.0 \\
\hline Smoke, No. (\%) & $32(30.5)$ & $12(34.3)$ & $20(28.4)$ & 0.553 \\
\hline \multicolumn{5}{|l|}{ Medication exposures prior to developing $\mathrm{CDI}^{\mathbf{b}}$, No. (\%) } \\
\hline Proton pump inhibitor & $49(46.7)$ & $21(60)$ & $28(40)$ & 0.054 \\
\hline Antiviral treatment & $47(44.8)$ & $19(54.3)$ & $28(40)$ & 0.168 \\
\hline Use of any antibiotic not directed at CDI & $46(43.8)$ & $24(68.6)$ & $22(31.4)$ & 0.000 \\
\hline Cephalosporin & $19(18.1)$ & $14(40)$ & $5(7.1)$ & 0.000 \\
\hline Carbapenem & $12(11.4)$ & $7(20)$ & $5(7.1)$ & 0.052 \\
\hline Fluoroquinlones & $13(12.4)$ & $9(25.7)$ & $4(5.7)$ & 0.003 \\
\hline$\beta$-lactam $/ \beta$-lactamase inhibitor combinations & $21(20)$ & $8(22.9)$ & $13(18.6)$ & 0.609 \\
\hline Other & $9(8.6)$ & $7(20)$ & $2(2.9)$ & 0.003 \\
\hline Antifungal agent & $5(4.8)$ & $3(8.6)$ & $2(2.9)$ & 0.199 \\
\hline \multicolumn{5}{|l|}{ Comorbidities, No. (\%) } \\
\hline Respiratory disease & $10(9.5)$ & $8(22.9)$ & $2(2.9)$ & 0.001 \\
\hline Cardiovascular disease & $16(15.2)$ & $6(17.1)$ & $10(14.3)$ & 0.704 \\
\hline Gastrointestinal disease & $14(13.3)$ & $8(22.9)$ & $6(8.6)$ & 0.043 \\
\hline Metabolic disorder & $10(9.5)$ & $5(14.3)$ & $5(7.1)$ & 0.244 \\
\hline Malignancy & $17(16.2)$ & $13(37.1)$ & $4(5.7)$ & 0.000 \\
\hline Prior hospitalization, No. (\%) & $56(53.3)$ & $17(48.6)$ & $39(55.7)$ & 0.494 \\
\hline Length of hospital stay & $14(18)$ & $25(24)$ & $12(12.25)$ & 0.000 \\
\hline Infection concomitant to CDI, No. (\%) & $14(13.3)$ & $8(22.9)$ & $6(8.6)$ & 0.043 \\
\hline Pulmonary infection concomitant to CDI, No. (\%) & $8(7.6)$ & $4(11.4)$ & $4(5.7)$ & 0.303 \\
\hline Leukocyte, cells $\times 10^{9} / \mathrm{L}$ & $5.4(3.5)$ & $7.4(5.9)$ & $5(2.5)$ & 0.000 \\
\hline Haemoglobin, g/dL & $130(35)$ & $118(34)$ & $136(31.5)$ & 0.001 \\
\hline Platelet, $\mathbf{1 0}^{9} / \mathrm{L}$ & $129(76)$ & $123(136)$ & $129(86.75)$ & 0.137 \\
\hline Albumin, $\mathbf{g} / \mathbf{d L}$ & $33.1(8.9)$ & $32.95(9.975)$ & $34.5(7.4)$ & 0.251 \\
\hline Creatinine, $\mu \mathrm{mol} / \mathrm{L}$ & $65(23)$ & $64(36)$ & $66.5(21)$ & 0.073 \\
\hline ALT, IU/L & $316(299)$ & $135(70)$ & $407(402)$ & 0.005 \\
\hline AST IU/L & $211(225)$ & $157(72)$ & $238(248)$ & 0.269 \\
\hline $\mathbf{T B}, \mu \mathrm{mol} / \mathbf{L}$ & $117(166.75)$ & $87(56)$ & $132(225)$ & 0.121 \\
\hline INR & $1.2(0.46)$ & $1.13(0.36)$ & $1.27(0.60)$ & 0.195 \\
\hline PT & $13.9(5.8)$ & $12.9(4.7)$ & $14.5(7.05)$ & 0.093 \\
\hline Death, No. (\%) & $6(5.7)$ & $2(5.7)$ & $4(5.7)$ & 1.000 \\
\hline
\end{tabular}

Abbreviations: ALT, alanine aminotransferase; AST, aspartate transaminase; CDI, Clostridium difficile infection; INR, international normalized ratio; PT, prothrombin time; $\mathrm{TB}$, total bilirubin.

${ }^{\mathrm{a}}$ Data were expressed as mean \pm standard deviation (SD), median (IQR), or No. (\%).

${ }^{\mathrm{b}}$ Comparisons between the CHB and control groups were performed by Student t-test, Mann-Whitney's U-test, or chi-square test. 


\subsection{Clinical Features and Microbiological Findings}

As shown in Table 1, there were significant differences in prior antibiotic exposure, prolonged hospital stay, high leukocyte cell count, hemoglobin, alanine aminotransferase (ALT) level, respiratory disease, gastrointestinal disease, malignancy, and high Charlson score between the CDI and control groups $(\mathrm{P}<0.05)$. The prior antibiotic exposure was $68.6 \%$ in the CDI group, while this was $31.4 \%$ in the control group. As noted, both cephalosporin and fluoroquinolones were more widely used in the CDI group, when compared with the control group. Furthermore, no correlation was found between proton pump inhibitors or antivirals and CDI status ( $\mathrm{P}>0.05)$.

Patients with $C$. difficile infection had more comorbidities and higher Charlson scores than subjects in the control group. Major concomitant diseases, including malignancy, gastrointestinal disease, and respiratory disease, were more frequent in $\mathrm{CHB}$ patients with CDI than the control group. A total of 35 toxigenic $C$. difficile strains were analyzed by MLST, and these were assigned to 15 different STs. Four isolates (28.1\%) had A-B + CDT-strains, while 30 (85.7\%) isolates had A+B + CDT - strains. One (2.9\%) isolate had A + $\mathrm{B}+\mathrm{CDT}+$ strains (ST-5). ST-35 and ST-3 both accounted for 23.3 (7/30) of the A + B + CDT- strains. Three of the A-B+CDTstrains were classified as ST-37, while one was classified as ST-39.

\subsection{Risk Factors}

Risk factors were analyzed and extracted by comparing 35 CDI patients and 70 matched control subjects by univariate and step-wise multivariate analysis (Tables 1 and 2). Prolonged hospital stay (OR: 1.045; 95\% CI: 1.006 to 1.086 ) and higher Charlson score on admission (OR:3.063; 95\% CI: 1.602 to 5.857) were found to be independent factors for the development of CDI among CHB patients without cirrhosis.

\section{Discussion}

In the present study, CDI was investigated in a cohort of 105 non-cirrhotic CHB patients. It was found that 33\% $(n=35)$ of these patients developed CDI during their hospital stay. The identified risk factors included prolonged hospital stay and higher Charlson score, which made patients with CHB more susceptible to CDI. The morbidity and mortality of CDI have steadily increased in Europe and America (14-16). As reported, CDI-affected populations have become larger than that of methicillin-resistant Staphylococcus aureus (MRSA), and an estimated $12.1 \%$ of all health care-associated infections were attributed to CDI in America (17). Liver disease is a known risk for CDI (5). This study found that 33\% of CHB patients became infected with $\mathrm{CD}$ during hospitalization, which was higher than the general population (3). In the last few decades, the clinical management of hepatitis B has mainly focused on antiviral treatment. This often overlooks the negative impact of chronic liver injury on intestinal flora $(18,19)$, which may lead to an imbalanced microbiota in the gut that could favor $C$. difficile colonization and infection, or other life-threatening complications (9).

In the present study, the median hospital stay of patients in the CDI group was two times longer than that of subjects in the control group. Spores of $C$. difficile may survive even in harsh environments. The $C$. difficile contaminated environment in health care facilities and patients colonized or infected with $C$. difficile serve as important sources of hospital-based transmission (20). It was confirmed that patients in the CDI group had a prolonged hospital stay. In a previous study, ST-35 and ST-3 were among the most prevalent types in the hospital (21), and were the two predominant types in this study. The current researchers speculated that transmission events may exist, although discriminatory bacterial typing techniques, such as whole genome sequencing, are needed to validate these findings.

The inappropriate use of any antibiotics could trigger C. difficile colonization and infection (22). Cephalosporin and fluoroquinolones, which display a broad antibacterial spectrum and are potent, were most frequently used in this cohort. The inappropriate use of fluoroquinolones triggered several outbreaks of CDI in North America and Europe (23). The problem with the inappropriate use of antibiotics is more severe in China (24). The current research found that both cephalosporin and fluoroquinolones were more widely used in the CDI group, which may make CHB patients more susceptible to CDI. Antibiotics could disturb the flora equilibrium in the gastrointestinal tract, facilitating $C$. difficile spores to colonize, replicate and produce toxins. In addition, changes in the intestinal mucosa barrier in patients with CHB induced gastrointestinal microbial products of bacterial peptidoglycan, flagellin and metabolic byproducts easier to aggravate (9). The investigators previously noted that the peak of CDI incidence in the hospital of the current study occurred in internal medicine wards, where patients with various chronic diseases had longer hospital stays (22).

The present study had several limitations. First, the number of enrolled patients was relatively small. One of the reasons for such a small number was that physicians, who lacked CDI awareness, frequently overlooked the possibility of CDI among patients with unexplained diarrhea, leading to only $35 \mathrm{CHB}$ patients diagnosed with concomitant CDI in nearly six years at the hospital. On the other 


\begin{tabular}{|c|c|c|c|c|}
\hline \multirow[t]{2}{*}{ Variable } & \multicolumn{2}{|c|}{ Univariate Analysis } & \multicolumn{2}{|c|}{ Multivariate Analysis } \\
\hline & OR $(95 \% \mathrm{CI})$ & PValue & $\mathrm{OR}(95 \% \mathrm{CI})$ & P Value \\
\hline Age & $1.011(0.980-1.042)$ & 0.487 & & \\
\hline Use of any antibiotic not directed at CDI & $4.760(1.986-11.408)$ & 0.000 & & \\
\hline Hospitalization & $1.057(1.022-1.094)$ & 0.001 & $1.045(1.006-1.086)$ & 0.023 \\
\hline Infection concomitant to CDI & $3.160(1.001-9.982)$ & 0.050 & & \\
\hline Charlson comorbidity index score & $3.733(2.143-6.502)$ & 0.000 & $3.063(1.602-5.857)$ & 0.001 \\
\hline Respiratory disease & $10.074(2.009-50.517)$ & 0.005 & & \\
\hline Gastrointestinal disease & $3.160(1.001-9.982)$ & 0.050 & & \\
\hline Malignancy & $9.750(2.878-33.029)$ & 0.000 & & \\
\hline Leukocyte, cells $\times 10^{9} / \mathrm{L}$ & $1.340(1.136-1.580)$ & 0.001 & & \\
\hline Hemoglobin, $g / d L$ & $0.968(0.949-0.988)$ & 0.002 & & \\
\hline ALT & $0.997(0.995-0.000)$ & 0.010 & & \\
\hline
\end{tabular}

Abbreviations: ALT, alanine aminotransferase; CDI, Clostridium difficile infection; CI, confidence interval OR, odds ratio.

hand, the current research may have underestimated the incidence rate of CDI in CHB patients. Second, this was a single-center study, and the present findings must be verified in future multicenter-based studies.

\subsection{Conclusions}

It was found that $33 \%$ of admitted $\mathrm{CHB}$ patients acquired CDI during their hospital stay. Both longer hospital stay and higher Charlson score on admission represented risk factors for the acquisition of CDI among hospitalized $\mathrm{CHB}$ patients. These risk factors may alert physicians to become aware of the possibility of CDI, and to actively seek for specific pathogen detection for early diagnosis and timely treatment. Furthermore, greater emphasis on infection control measures and antimicrobial stewardship in CHB patients during hospital admission is needed, and these efforts may reduce incident rate of CDI in these patients.

\section{Footnotes}

Authors' Contribution: Study concept and design, Lanjuan Li; analysis and interpretation of data, Yunbo Chen, Tao Lv, Silan Gu, Ping Shen, Yunhui Fang and Jiazheng Quan; drafting of the manuscript, Yunbo Chen and Qiaodi Gui; critical revision of the manuscript for important intellectual content, Guangyong Ye; statistical analysis, Qiaomai Xu. Yunbo Chen and Qiaodi Gui contributed equally to this work.

Conflict of Interests: The authors declared that they had no conflicts of interest regarding this work.
Funding/Support: This work was supported by Key research and development program of Zhejiang province (grant no. 2015C03032) and the independent foundation of the State Key Laboratory for Diagnosis and Treatment of Infectious Diseases, The First Affiliated Hospital of Medical College, Zhejiang University (grant no. 2016ZZ04).

\section{References}

1. Bartlett JG, Moon N, Chang TW, Taylor N, Onderdonk AB. Role of Clostridium difficile in antibiotic-associated pseudomembranous colitis. Gastroenterology. 1978;75(5):778-82. [PubMed: 700321].

2. Warny M, Pepin J, Fang A, Killgore G, Thompson A, Brazier J, et al. Toxin production by an emerging strain of Clostridium difficile associated with outbreaks of severe disease in North America and Europe. Lancet. 2005;366(9491):1079-84. doi: 10.1016/S0140-6736(05)67420-X. [PubMed: 16182895].

3. Leffler DA, Lamont JT. Clostridium difficile infection. NEnglJMed. 2015 Apr 16;372(16):1539-48. doi: 10.1056/NEJMra1403772.

4. Zimlichman E, Henderson D, Tamir O, Franz C, Song P, Yamin CK, et al. Health care-associated infections: a meta-analysis of costs and financial impact on the US health care system. JAMA Intern Med. 2013;173(22):2039-46. doi: 10.1001/jamainternmed.2013.9763. [PubMed: 23999949].

5. Trifan A, Stoica O, Stanciu C, Cojocariu C, Singeap AM, Girleanu I, et al. Clostridium difficile infection in patients with liver disease: a review. Eur J Clin Microbiol Infect Dis. 2015;34(12):2313-24. doi: 10.1007/s10096015-2501-z. [PubMed: 26440041].

6. Vanjak D, Girault G, Branger C, Rufat P, Valla DC, Fantin B. Risk factors for Clostridium difficile infection in a hepatology ward. Infect Control Hosp Epidemiol. 2007;28(2):202-4. doi: 10.1086/511790. [PubMed: 17265403].

7. Ali M, Ananthakrishnan AN, Ahmad S, Kumar N, Kumar G, Saeian K. Clostridium difficile infection in hospitalized liver transplant patients: a nationwide analysis. Liver Transpl. 2012;18(8):9728. doi: 10.1002/lt.23449. [PubMed: 22505356]. [PubMed Central: PMC3405162]. 
8. Mittal C, Hassan S, Arshad S, Jeepalyam S, Bruni S, Miceli M, et al. Clostridium difficile infection in liver transplant recipients: a retrospective study of rates, risk factors and outcomes. Am J Transplant. 2014;14(8):1901-7. doi:10.1111/ajt.12798. [PubMed: 24902610].

9. Lu H, Wu Z, Xu W, Yang J, Chen Y, Li L. Intestinal microbiota was assessed in cirrhotic patients with hepatitis B virus infection. Intestinal microbiota of HBV cirrhotic patients. Microb Ecol. 2011;61(3):693-703. doi: 10.1007/s00248-010-9801-8. [PubMed: 21286703].

10. Steele SR, McCormick J, Melton GB, Paquette I, Rivadeneira DE, Stewart D, et al. Practice parameters for the management of Clostridium difficile infection. Dis Colon Rectum. 2015;58(1):10-24. doi: 10.1097/DCR.0000000000000289. [PubMed: 25489690].

11. Kato H, Kato N, Watanabe K, Iwai N, Nakamura H, Yamamoto T, et al. Identification of toxin A-negative, toxin B-positive Clostridium difficile by PCR. J Clin Microbiol. 1998;36(8):2178-82. [PubMed: 9665986]. [PubMed Central: PMC105000].

12. Stubbs S, Rupnik M, Gibert M, Brazier J, Duerden B, Popoff M. Production of actin-specific ADP-ribosyltransferase (binary toxin) by strains of Clostridium difficile. FEMS Microbiol Lett. 2000;186(2):307-12. doi: 10.1111/j.1574-6968.2000.tb09122.x. [PubMed: 10802189].

13. Griffiths D, Fawley W, Kachrimanidou M, Bowden R, Crook DW, Fung R, et al. Multilocus sequence typing of Clostridium difficile. Clin Microbiol. 2010;48(3):770-8. doi: 10.1128/JCM.01796-09. [PubMed: 20042623]. [PubMed Central: PMC2832416].

14. Hensgens MP, Goorhuis A, Dekkers OM, van Benthem BH, Kuijper EJ. All-cause and disease-specific mortality in hospitalized patients with Clostridium difficile infection: a multicenter cohort study. Clin Infect Dis. 2013;56(8):1108-16. doi:10.1093/cid/cis1209. [PubMed: 23300235].

15. Bauer MP, Notermans DW, van Benthem BH, Brazier JS, Wilcox MH, Rupnik M, et al. Clostridium difficile infection in Europe: A hospital-based survey. Lancet. 2011;377(9759):63-73. doi:10.1016/S01406736(10)61266-4. [PubMed: 21084111].

16. Redelings MD, Sorvillo F, Mascola L. Increase in Clostridium difficilerelated mortality rates, United States, 1999-2004. Emerg Infect Dis. 2007;13(9):1417-9. doi: 10.3201/eid1309.061116. [PubMed: 18252127]. [PubMed Central: PMC2857309].
17. Magill SS, Edwards JR, Bamberg W, Beldavs ZG, Dumyati G, Kainer MA, et al. Multistate point-prevalence survey of health care-associated infections. N Engl J Med. 2014;370(13):1198-208. doi: 10.1056/NEJMoa1306801. [PubMed: 24670166]. [PubMed Central: PMC4648343].

18. Beckebaum S, Malago M, Dirsch O, Cicinnati VR, Trippler M, Lampertico P, et al. Efficacy of combined lamivudine and adefovir dipivoxil treatment for severe HBV graft reinfection after living donor liver transplantation. Clin Transplant. 2003;17(6):554-9. doi:10.1046/j.09020063.2003.00097.x. [PubMed:14756274].

19. Castelnau C, Le Gal F, Ripault MP, Gordien E, Martinot-Peignoux M, Boyer N, et al. Efficacy of peginterferon alpha-2b in chronic hepatitis delta: relevance of quantitative RT-PCR for follow-up. Hepatology. 2006;44(3):728-35. doi:10.1002/hep.21325. [PubMed: 16941695].

20. Vonberg RP, Kuijper EJ, Wilcox MH, Barbut F, Tull P, Gastmeier P, et al. Infection control measures to limit the spread of Clostridium difficile. Clin Microbiol Infect. 2008;14 Suppl 5:2-20. doi: 10.1111/j.14690691.2008.01992.x. [PubMed: 18412710].

21. Chen YB, Gu SL, Shen P, LvT, Fang YH, Tang LL, et al. Molecular epidemiology and antimicrobial susceptibility of Clostridium difficile isolated from hospitals during a 4-year period in China. J Med Microbiol. 2018;67(1):52-9. doi: 10.1099/jmm.0.000646. [PubMed: 29160203].

22. Xu Q, Chen Y, Gu S, Lv T, Zheng B, Shen P, et al. Hospital-acquired Clostridium difficile infection in Mainland China: A seven-year(20092016) retrospective study in a large university hospital. Sci Rep. 2017;7(1):9645. doi: 10.1038/s41598-017-09961-0. [PubMed: 28852010]. [PubMed Central: PMC5575102].

23. Abou Chakra CN, Pepin J, Sirard S, Valiquette L. Risk factors for recurrence, complications and mortality in Clostridium difficile infection: a systematic review. PLoS One. 2014;9(6). e98400. doi: 10.1371/journal.pone.0098400. [PubMed: 24897375]. [PubMed Central: PMC4045753].

24. Tang C, Cui L, Xu Y, Xie L, Sun P, Liu C, et al. The incidence and drug resistance of Clostridium difficile infection in Mainland China: a systematic review and meta-analysis. Sci Rep. 2016;6:37865. doi: 10.1038/srep37865. [PubMed: 27897206]. [PubMed Central: PMC5126672]. 